\title{
Asymptotic Methods in the Nonlinear Mean-field Dynamo
}

\author{
D.D. Sokoloff ${ }^{1}$, A. Shukurov ${ }^{2}$ and A.A. Ruzmaikin ${ }^{2}$ \\ ${ }^{1}$ Physics Department, Moscow State University, Moscow, USSR \\ ${ }^{2}$ IZMIRAN, USSR Academy of Sciences, Troitsk, Moscow Region, USSR
}

\begin{abstract}
We discuss the methods and results of analysis of nonlinear mean-field dynamo models based on $\alpha$-quenching in two asymptotic regimes, namely for weakly and highly supercritical excitation. In the former case the spatial distribution of the steady-state magnetic field is close to that given by the neutrally stable eigenfunction of the corresponding kinematic dynamo. In the latter case the magnetic field distribution within the main part of the dynamo volume is presumably determined by the balance between the Lorentz and Coriolis forces while near the boundaries boundary layers arise in which the field adjusts itself to the boundary conditions. The asymptotic behaviour of the highly supercritical $\alpha \omega$-dynamos is sensitive to the particular form of dependence of the mean helicity on magnetic field while $\alpha^{2}$-dynamos are less sensitive to this dependence.
\end{abstract}

\section{Introduction}

One of widely employed approaches to the analysis of the steady states of magnetic field generated by hydromagnetic mean-field dynamos is based on introduction of the mean helicity as an explicit function of the large-scale magnetic field. In fact, this approach is an extrapolation to nonlinear regime of ideas of the kinematic dynamo theory in which the velocity field is prescribed as a known function of position whose form is justified by simple physical arguments of a general character, rather than follows from the Navier-Stokes equations. Such approach is much less complicated than solution of the coupled induction and Navier-Stokes equations and allows the analysis of general types of evolution in nonlinear dynamo systems and also a qualitative comparison of the theory with observations.

Two basic concepts of the steady-state distribution of magnetic field are usually discussed in the literature. It is often presumed that this distribution is close to the neutrally stable eigenfunction of the kinematic dynamo based on the observed (or presumed) velocity field. On the other hand, comparisons with observational results sometimes rely on the assumption either of equipartition of kinetic and magnetic energies or of local balance between the Lorentz and Coriolis forces. Our 
results discussed below allow the reconciliation of these apparently contradictory ideas.

\section{The nature of nonlinear solutions}

A steady state of a dynamo arises when the generated magnetic field modifies the velocity field in such way that the generation efficiency becomes marginally weak due to, e.g., a local balance of amplification and dissipation. In this state the effective dynamo number (or any other relevant measure of the generation efficiency) is presumed to decrease down to the critical value $D_{0}$ which corresponds to vanishing of the field growth rate in the allied kinematic dynamo. These arguments contain an implicit contradiction: on the one hand, it is presumed that modification of the velocity field has a local character and, on the other hand, the criterion of such balance is based on a global parameter, the dynamo number.

A resolution of this contradiction follows from the fact that the generated magnetic field is inhomogeneous and, therefore, the velocity field is modified to different extent at different positions. Meanwhile, the critical dynamo number is rather sensitive to the form of the velocity field and, thereby, the effective critical dynamo number cannot be determined basing on the initial velocity field and remains unknown in advance. It is clear that the steady-state magnetic field is stronger in those regions where the fluid density or kinetic energy density is larger. Correspondingly, the velocity field is modified more strongly in these regions.

The arguments of the first paragraph in this section bring us to the conclusion that the steady-state distribution of magnetic field is close to the neutrally stable eigenfunction of the corresponding kinematic dynamo. Meanwhile, the arguments of the second paragraph imply that spatial distributions of both the velocity field and the fluid density affect spatial distribution of the steady-state magnetic field, i.e. one comes to an idea of a balance between kinetic and magnetic energies or between Lorentz and Coriolis forces in the steady state of the dynamo. Although these two ideas on the nature of the steady state - the neutrally stable eigenfunction and the dynamic equilibrium - seem to be inconsistent with each other, they both exist independently in the current literature.

The aim of this contribution is to demonstrate that both nonlinear regimes can be realized in dynamo systems. In the case of weakly supercritical excitation, $\left|D-D_{0}\right| \ll\left|D_{0}\right|$ with $D$ the dynamo number, the steady-state distribution of magnetic field is asymptotically close to the neutrally stable eigenfunction while the maximal field strength is proportional to $\left|D-D_{0}\right|^{1 / 2}$. In the other limiting case of highly supercritical excitation, $|D| \gg\left|D_{0}\right|$, the magnetic field is distributed in accordance with the local balance between the Lorentz and Coriolis forces.

For definiteness, we employ the terminology of the mean-field $\alpha \omega$-dynamos although our arguments are applicable in a general case. For illustration, we consider two particular forms of the helicity coefficient $\alpha(\boldsymbol{r})$ as a function of magnetic field: 


$$
\alpha(\boldsymbol{r}, \boldsymbol{B})=\alpha_{0}(\boldsymbol{r})\left[1-g(\boldsymbol{r})|\boldsymbol{B}|^{2}\right],
$$

and

$$
\alpha(\boldsymbol{r}, \boldsymbol{B})=\frac{\alpha_{0}(\boldsymbol{r})}{1+g(\boldsymbol{r})|B|^{2}},
$$

where $B$ is the large-scale magnetic field and $\alpha_{0}(r)$ and $g(r)$ are certain functions of position. We consider the $\alpha$-quenching as the only nonlinearity although $\omega$ quenching can be considered in the same way.

The physical meaning of the function $g(\boldsymbol{r})$ is the following. This function is connected with the characteristic strength of magnetic field which is determined, in turn, by a specific physical mechanism of saturation of the dynamo. For example, such saturation in the mean-field dynamos can be shown to be associated with a balance of the Lorentz and Coriolis forces which is formally similar to the magnetostrophic balance typical of convective dynamos:

$$
\frac{\tilde{B}_{\phi} \tilde{B}_{r}}{4 \pi L} \simeq \rho v \Omega
$$

where $\tilde{B}_{\phi}$ and $\tilde{B}_{r}$ are the characteristic azimuthal and radial cylindrical components of the large-scale magnetic field, respectively, $L$ is the field scale, $v$ is the r.m.s. turbulent velocity and $\Omega$ is the overall angular velocity of rotation. Then the function $g(\boldsymbol{r})$ is defined as $g(\boldsymbol{r})=|\tilde{\boldsymbol{B}}|^{-2}$, where $\tilde{B}$ follows from the balance relation (3).

\section{The form of nonlinear solutions}

In this section we discuss the method of derivation and the explicit form of nonlinear solutions of the mean-field dynamo problem in the two limiting cases mentioned above.

\subsection{Weakly supercritical dynamos}

In the case when the dynamo number $D$ only slightly exceeds the critical value $D_{0}$, i.e. $\left|D-D_{0}\right| \ll\left|D_{0}\right|$, the steady-state strength of magnetic field is rather small and the mean helicity is modified only weakly. Therefore, the steady-state distribution of the helicity can be expected to be close to that corresponding to $\boldsymbol{B}=0$, i.e. $\alpha(\boldsymbol{r}) \approx \alpha_{0}(\boldsymbol{r})$. As a result, the spatial distribution of magnetic field is asymptotically close to that given by the neutrally stable eigenfunction of the kinematic dynamo with $\alpha(\boldsymbol{r})=\alpha_{0}(\boldsymbol{r})$. The corresponding nonlinear asymptotic solutions have been obtained by Rüdiger (1973) for the simplest case of the $\alpha^{2}$ dynamo and by Kvasz et al. (1990) for the $\alpha \omega$-dynamo, both for a nonlinearity of the form given by eq. (1) and for a thin-disk geometry. For $\mid D-D)|\ll| D_{0} \mid$ we have $|\boldsymbol{B}|=O\left(\left|D-D_{0}\right|^{1 / 2}\right) \ll 1$ and, therefore, any dependence of $\alpha$ on $\boldsymbol{B}$ (e.g., 
that given by eq. 2) can be expanded in power series in $B^{2}$ and the form (1) is a general one. Kleeorin and Ruzmaikin (1981) have considered nonlinear weakly supercritical solutions for $\alpha$ described by a differential equation rather that by an algebraic function.

\subsection{Highly supercritical dynamos}

One-dimensional highly supercritical $\alpha^{2}$-dynamos have been recently investigated numerically by Meinel and Brandenburg (1990) (see also Baryshnikova and Shukurov, 1987). An analytical asymptotic analysis of a one-dimensional $\alpha \omega-$ dynamo with nonlinearity of the form (1) has been performed by Kvasz et al. (1990) whose results and their generalizations are discussed below.

The stationary $\alpha \omega$-dynamo equations in a slab surrounded by vacuum can be reduced to the following equation for $B$, the $y$-component (or the azimuthal one in cylindrical coordinates) of the large-scale magnetic field (it is presumed that $|B| \approx B$ which is typical of $\alpha \omega$-dynamos):

$$
\frac{\mathrm{d}^{3} B}{\mathrm{~d} z^{3}}+D \alpha_{0}(z)\left[1-g(z) B^{2}\right] B=0
$$

with the boundary conditions $B(1)=\mathrm{d}^{2} B(1) / \mathrm{d} z^{2}=\mathrm{d} B(0) / \mathrm{d} z=0$, where $z$ is the coordinate measured across the slab (Vainshtein and Ruzmaikin, 1972) and the nonlinearity is chosen to be of the form (1).

For $|D| \gg\left|D_{0}\right|$ eq. (4) represents a singularly perturbed equation and its solution reduces to a combination of boundary layers situated near $|z|=1$ (the slab surface) and, possibly, $z=0$ (the midplane) and the smooth (degenerate) field distribution in the main part of the slab, for which the field derivative can be neglected to the leading order. The structure of the boundary layers is considered in detail by Kvasz et al. (1990). Here we note that their thickness is as small as $O\left(|D|^{-1 / 3}\right)$ and they hardly can be observed in real dynamo objects. Therefore, here we concentrate on the degenerate solution.

For $|D| \gg 1$ we have $B=O(1)$ and $\mathrm{d}^{3} B / \mathrm{d} z^{3}=O(1)$ outside the boundary layers and eq. (4) can be satisfied when $1-g(z) B^{2}=O\left(|D|^{-1}\right)$. Thus, the degenerate solution is given by $B(z)=[g(z)]^{-1 / 2}+O\left(|D|^{-1}\right)$. The terms of the order of $|D|^{-1}$ and higher ones can be easily obtained using the regular perturbation techniques (Kvasz et al., 1990). Thus, for a nonlinearity of the form (1) the field distribution outside the boundary layers does not depend, to the leading order, on the magnetic diffusivity.

For $\alpha(r, B)$ of the form (2), the asymptotic solution has somewhat different properties. In this case eq. (4) is replaced by

$$
\frac{\mathrm{d}^{3} B}{\mathrm{~d} z^{3}}+D \frac{\alpha_{0}(z)}{1+g(z) B^{2}} B=0 .
$$

In contrast to eq. (4), the second term on the left-hand side of eq. (5) cannot be identically zero. Therefore, the field strength in the steady state is large, $B \gg 1$ (in contrast to eq. (4) where $B=O(1)$ ). As a result, the balance of the field generation 
and diffusion is maintained even at the leading order and the derivative in eq. (5) cannot be neglected even outside the boundary layers. One can easily verify that in this case $B=O\left(|D|^{1 / 2}\right)$. The leading-order solution is given by $B(z)=|D|^{1 / 2} b(z)$ where $b(z)$ obeys the following equation:

$$
b \frac{\mathrm{d}^{3} b}{\mathrm{~d} z^{3}}+\frac{\alpha_{0}(z)}{g(z)}=0 .
$$

Notice that this solution depends on magnetic diffusivity through the dynamo number $D$.

Thus, properties of highly supercritical $\alpha \omega$-dynamos are very sensitive to the particular form of nonlinearity. The case of the $\alpha^{2}$-dynamo is much simpler in this respect because the highest order derivative can be always neglected outside the boundary layers in equation

$$
\frac{\mathrm{d}^{2} B}{\mathrm{~d} z^{2}}-\mathrm{i} R_{\alpha} \frac{\mathrm{d}}{\mathrm{d} z}[\alpha(z, B) B]=0,
$$

which corresponds to eqs (4) or (5), and the degenerate solution governed by $\alpha(z, B) B=$ const always exist. This implies that, in contrast to $\alpha \omega$-dynamos, solutions of highly supercritical $\alpha^{2}$-dynamos depend only weakly on the magnetic diffusivity.

\section{References}

Baryshnikova, Yu.S., Shukurov, A.: 1987, Astron. Nachr. 308, 89

Kleeorin, N.I., Ruzmaikin, A.A.: 1981, Geophys. Astrophys. Fluid Dyn. 17, 281

Kvasz, L., Sokoloff, D., Shukurov, A.: 1990, Geophys. Astrophys. Fluid Dyn. in press

Meinel, R., Brandenburg, A.: 1990, Astron. Astrophys., in press

Rüdiger, G.: 1973, Astron. Nachr. 294, 183

Vainshtein, S.I., Ruzmaikin, A.A.: 1972, Sov. Astron. 16, 365 